\title{
Die Union für das Mittelmeer: Ambitionen und Realität - eine ernüchternde Zwischenbilanz der französisch- ägyptischen Präsidentschaft
}

\author{
Thomas Demmelhuber und Andreas Marchetti*
}

Das von der französischen Regierung initiierte Gipfeltreffen euro-mediterraner Staatsund Regierungschefs in Paris am 13. Juli 2008 und die damit verbundene Ergänzung bisheriger euro-mediterraner Politikinitiativen weckten Hoffnungen sowohl in Europa als auch in den Partnerstaaten im südlichen Mittelmeerraum. Eine Verringerung der weithin kritisierten Divergenz zwischen Anspruch und Wirklichkeit der Mittelmeerpolitik der Europäischen Union schien greifbar zu sein. Institutionelle Veränderungen, die damit korrelierende Stärkung des Partnerschaftsgedankens und präzisere Felder der Kooperation sollten zu einer Intensivierung ${ }^{1}$ und damit letztlich auch zu einer Depolitisierung der Kooperation führen. Umstrittene Politikfelder, vor allem in der politischen und sicherheitspolitischen Zusammenarbeit, blieben im Gründungsdokument der Union für das Mittelmeer (UfM) ${ }^{2}$ vom Juli 2008 zwar berücksichtigt, in der praktischen Politikformulierung der Projektbezogenheit aber nachgeordnet. Ferner fand auch das Prinzip der , variablen Geometrie', das heißt die Möglichkeit projektspezifisch über Teilnahme oder Nichtteilnahme zu entscheiden, Einzug in das Schlussdokument von Paris. Damit sollte durch eine Abkopplung vom Prinzip der Einstimmigkeit eine konstruktive Zusammenarbeit zwischen kooperationswilligen Partnerstaaten erleichtert werden. ${ }^{3}$

Dennoch, mehr als zweieinhalb Jahre nach der Gründung zeigt sich dem Betrachter eine sehr schleppende Umsetzung der vereinbarten institutionellen Neuerungen und inhaltlichen Neuakzentuierung. Zudem fällt eine nicht antizipierte Politisierung der Kooperation auf, die den Grundannahmen der UfM zur Vertiefung euro-mediterraner Kooperation zuwiderläuft. Der vorliegende Beitrag fragt daher nach den Hintergründen dieser verstärkten Politisierung trotz gegenläufiger Intention. Dabei ist unter Politisierung vor allem der verstärkte Widerhall etablierter Konfliktlinien im euro-mediterranen Verbund in inhaltlich mit diesen zunächst nicht verknüpften Themen- und Kooperationsfeldern zu verstehen, wohingegen die erstrebte Depolitisierung besonders den Wunsch nach pragmatischen Fortschritten vor dem Hintergrund einer Neugewichtung im Zielgefüge europäischer Mittelmeerpolitik reflektiert. Diese sollte den Akzent von einem wenig erfolgreichen normativen Ansatz hin zu einer strategischen und stabilitätsorientierten Politikausrichtung verlagern. Dabei ist angesichts der Heterogenität der UfM-Partnerstaaten und dem weiterhin ungelösten Nahostkonflikt, die unter anderem für ein grundsätzlich hohes Politisierungsniveau in den Beziehungen verant-

* Dr. Thomas Demmelhuber, Institut für Politische Wissenschaft, Friedrich-Alexander-Universität ErlangenNürnberg.

Dr. Andreas Marchetti, Zentrum für Europäische Integrationsforschung (ZEI), Universität Bonn.

1 So auch Forderungen aus dem südlichen Mittelmeerraum. Vgl. Ahmed Driss: North-African Perspectives, in: Roberto Aliboni/Ahmed Driss/Tobias Schumacher/Alfred Tovias: Putting the Mediterranean Union in Perspective, Euro-Mediterranean Study Commission: EuroMeSCo Paper 68, 2008, S. 19-23.

2 Joint Declaration of the Paris Summit for the Mediterranean. Paris 13 July 2008.

3 Dies folgt in Analogie zu den Möglichkeiten der Flexibilisierung innerhalb des Rahmens der Europäischen Union, wie sie erstmals im Vertrag von Amsterdam (1997) mit Bestimmungen zur Verstärkten Zusammenarbeit formalisiert wurden. 
wortlich sind, hervorzuheben, dass mit den Prozessbeschreibungen von Politisierung und Depolitisierung keine entsprechenden Zustandsbeschreibungen des euro-mediterranen Beziehungsgefüges einhergehen. Vielmehr gilt es zu prüfen, inwiefern die Beziehungen eher politisiert oder depolitisiert wurden. Gerade vor diesem Hintergrund ist zu fragen, ob es alleinig die Komplexität des Nahostkonflikts ist, welche das euro-mediterrane Beziehungsgeflecht deutlich politisiert und lähmt - ganz nach dem Motto, ,[c]e n'est pas l'argent qui manque. Ce qui manque, c'est la paix"4 - oder ob auch andere Faktoren hierbei eine Rolle spielen. Gibt es trotz eines letztlich nüchternen Befunds der Kooperation Felder, die seit Lancierung der UfM nachhaltige Impulse erfuhren? In welchem Kontext ist dabei die Rolle der Staaten Ägypten und Frankreich, welche die Co-Präsidentschaft der UfM innehaben, zu betrachten? Wie ist schließlich die bisherige aber auch künftige Entwicklung vor dem Hintergrund der gegenwärtigen fundamentalen Umwälzungen im Mittelmeerraum zu sehen?

\section{Die Europäische Union und ihre südlichen Nachbarn}

Die Europäische Union hat seit den Römischen Verträgen 1957 mit der Region des Nahen und Mittleren Ostens mehrdimensionale, in einzelne Subregionen differenzierte Kooperationsmechanismen aufgebaut. Mit Abstand am umfangreichsten institutionalisiert sind diese Beziehungsstränge mit den Staaten im südlichen Mittelmeerraum von Mauretanien und Marokko im Westen bis in die Levante. Die Türkei, obwohl Mitglied euro-mediterraner Politikinitiativen, ist dabei als Sonderfall zu betrachten, aufgrund des Status als Beitrittskandidat zur Europäischen Union seit Dezember 1999, mit dem seit Oktober 2005 über eine Aufnahme als Vollmitglied verhandelt wird. Rückblickend ist vor allem das Jahr 1995 als Zäsur der europäischen Mittelmeerpolitik zu sehen, da dieses Jahr für den Beginn eines umfassenden Politikansatzes gegenüber der Region steht. Internationale (Ende des Ost-WestKonflikts), regionale (anvisierte Osterweiterung der Europäischen Union und Fortschritte im Nahostfriedensprozess) und innereuropäisch-institutionelle Rahmenbedingungen (Vertrag von Maastricht) waren dabei die Grundvoraussetzungen für die formale Aufwertung des südlichen Mittelmeerraums in der außenpolitischen Politikformulierung der Europäischen Union. Ausgehend vom Gipfel euro-mediterraner Staats- und Regierungschefs in Barcelona im November 1995 wurde die Euro-Mediterrane Partnerschaft (EMP) gegründet. Anlässlich des Gipfels wurden drei inhaltliche Partnerschaften für die auch als Barcelona-Prozess bezeichnete Politikinitiative ausgewiesen: erstens die normativ aufgeladene politische und sicherheitspolitische Partnerschaft, zweitens die besonders auf die Errichtung einer euro-mediterranen Freihandelszone abzielende wirtschaftliche und finanzielle Partnerschaft sowie drittens die Partnerschaft in sozialen, kulturellen und menschlichen Belangen, welche neben den Regierungen der Partnerstaaten vor allem auch die Zivilgesellschaft in den MittelmeerDrittstaaten (MDL) einbinden möchte.

Seither erfuhr die Mittelmeerpolitik der Europäischen Union eine konzeptionelle und inhaltliche sowie unlängst mit der UfM auch geografische Erweiterung, ${ }^{5}$ durch die vor allem den Problemen in der zunächst schleppenden operativen Umsetzung der Politikinitiativen sowie neuen Bedrohungsszenarien Rechnung getragen wurde. Zentrale Etappen waren dabei die Inklusion des südlichen Mittelmeerraums in die Europäische Nachbarschaftspolitik (ENP) im Jahre 2004 und vier Jahre später auf französische Initiative die Errichtung der

4 Nicolas Sarkozy, zitiert in: EurActiv.fr: Union pour la Méditerranée: le financement des projets reste flou, 14.07.2008.

5 Die UfM umfasst nun 43 Mitglieder. Neu hinzu kamen Albanien, Kroatien, Montenegro, Bosnien-Herzegowina und das Fürstentum Monaco. 
UfM als Ergänzung beziehungsweise Fortentwicklung der EMP. ${ }^{6}$ Damit einher ging aber eine weitere Öffnung des maghrebinisch-levantinischen Mittelmeerraums in Richtung einer Inklusion des gesamten mediterranen Beckens, ungeachtet der geringen Homogenität der entsprechend unter einem Dach zusammengefassten Staaten. Bereits in den Beziehungen der Europäischen Union zu diesen wird dieser Befund deutlich: Neben den Assoziierungsabkommen mit den (meisten) Teilnehmern des Barcelona-Prozesses sind nunmehr auch die Beitrittskandidaten des Balkans und die Türkei einbezogen. Damit besteht neben ihrer Lage am Mittelmeer die einzige gemeinsame Eigenschaft dieser Drittstaaten darin, derzeit nicht Mitglied der Europäischen Union zu sein.

Frieden, Stabilität, Wohlstand und Sicherheit sind bis heute die Kernelemente der europäischen Interessensagenda im Mittelmeerraum, obgleich sich die Frage der Implementierung dieser Ziele in Kooperation mit den einzelnen MDL des Barcelona-Prozesses bis dato als schwierig erwies und die Europäische Union in Zielkonflikte in ihrer außenpolitischen Politikformulierung brachte. Einerseits verhindert die komplexe Interessenslage im arabisch-israelischen Konflikt eine regionale Vertiefung euro-mediterraner Beziehungen (Israel als gleichberechtigter Partner neben der Palästinensischen Autonomiebehörde und den weiteren arabischen MDL). Andererseits ist der begrenzte Erfolg europäischer Mittelmeerpolitik seit 1995 auch einem zentralen Zielkonflikt in der Außenpolitik der Europäischen Union geschuldet, die zum einen dem Selbstbild einer Wertegemeinschaft nach innen und nach auBen, ${ }^{7}$ zum anderen aber auch dem Rollenkonzept einer Sicherheits- und Stabilitätsgemeinschaft folgen möchte. So blieb in der praktischen Politikgestaltung die Einforderung demokratischer Mindeststandards gegenüber den mehrheitlich autoritären Herrschaftssystemen im südlichen Mittelmeerraum meist von nachgeordneter Bedeutung, da die Kooperation mit den herrschenden Eliten als prioritär erachtet wurde, um Sicherheitsrisiken in unmittelbarer Nachbarschaft wie zum Beispiel der illegalen Migration Rechnung zu tragen. Diese konzeptionellen Widersprüche der Mittelmeerpolitik sollte die UfM mit einer faktischen Ausklammerung konfliktgeladener Themenbereiche, flexibler Akteurskonstellationen entsprechend des Prinzips der variablen Geometrie und ipso facto mit einer Depolitisierung und dezidierten Projektbezogenheit der Kooperation verringern. Ausgangspunkt war dabei die Annahme der Stabilität der politischen Strukturen in den jeweiligen MDL, die sich seit den grundlegenden Umwälzungen zur Jahreswende 2010/2011 als trügerisch herausstellte. ${ }^{8}$

\section{Mittelmeerpolitik in Nord-Süd-Verantwortung}

Ein zentrales Element der UfM besteht darin, alle Anrainer des Mittelmeers gleichberechtigt unter dem Stichwort des Miteigentums (,co-ownership \%,joint ownership“) in die von Frankreich initiierte Neuerung der Mittelmeerpolitik einzubeziehen. Als Miteigentümer der UfM sollten die MDL auch zu Mitverantwortlichen werden. Damit sollte auch ihr Interesse an der Formulierung einer gemeinsamen Mittelmeerpolitik, die auch ihren Präferenzen

6 Vgl. dazu: Emanuel Adler/Federica Bicchi/Beverly Crawford/Raffaella del Sarto (Hrsg.): The Convergence of Civilizations: Constructing a Mediterranean Region, Toronto 2006; Thomas Demmelhuber: EU-Mittelmeerpolitik und der Reformprozess in Ägypten. Von der Partnerschaft zur Nachbarschaft, Baden-Baden 2009; Raffaella del Sarto/Tobias Schumacher: From EMP to ENP: What's at Stake with the European Neighbourhood Policy towards the Southern Mediterranean?, in: European Foreign Affairs Review 1/2005, S. 17-38.

7 Vgl. Michelle Pace: The Construction of EU Normative Power, in: Journal of Common Market Studies 5/2007, S. 1041-1064.

8 Vgl. zur trügerischen Stabilität, Andreas Marchetti: Promoting Good Governance. The Keystone to a Sustainable Mediterranean Policy, in: Andreas Marchetti (Hrsg.): Ten Years Euro-Mediterranean Partnership. Defining European Interests for the Next Decade, Zentrum für Europäische Integrationsforschung: ZEI Discussion Paper C 154, Bonn 2005, S. 47-58, hier S. 57-58. 
stärker Rechnung tragen soll, gestärkt werden. Den deutlichsten Ausdruck dieses Anspruchs einer Gleichberechtigung zwischen Nord und Süd, dem zuvor nur ansatzweise und implizit unter dem Stichwort der Partnerschaft Folge geleistet wurde, ${ }^{9}$ sollte die UfM in der Einrichtung einer zwischen ,Nord' (Europäische Union) und ,Süd' (MDL) aufgeteilten Präsidentschaft erfahren, worauf sich alle Beteiligten bereits im Vorfeld des Pariser Gipfels von 2008 verständigt hatten.

\section{Frankreichs vermeintliche Un-Ambition}

Die UfM geht auf die Initiative Nicolas Sarkozys zurück, der bereits als französischer Präsidentschaftskandidat im Februar 2007 in Toulon erste Ideen zur Einrichtung einer Mittelmeerunion vorstellte. ${ }^{10}$ Hiervon ausgehend wurde im Vorantreiben des Projekts unter Staatspräsident Sarkozy seitens des Élysée kontinuierlich auf das Scheitern des BarcelonaProzesses (,échec de Barcelone') verwiesen, weshalb eine neue Politikinitiative vonnöten sei. Ausgangspunkt der französischen Überlegungen war die Überzeugung von der Notwendigkeit, den politischen Ballast und die als zu ehrgeizig erachteten normativen Ambitionen des Barcelona-Prozesses abzuwerfen, um in pragmatischer und damit depolitisierender Absicht konkrete Fortschritte in den Beziehungen zu den MDL zu erzielen. ${ }^{11}$ Analog sollte der Türkei eine besondere Stellung in dieser Union zuteilwerden, um die Beitrittsambitionen ungeachtet bereits laufender Verhandlungen - umzulenken. Demgemäß vertrat Frankreich zunächst die Idee des ausschließlichen Einbezugs der unmittelbar betroffenen Staaten, das heißt der geografischen Mittelmeeranrainer.

Indessen regte sich gerade seitens nicht-mediterraner Mitgliedstaaten der Europäischen Union, allen voran Deutschland, Widerstand gegen diesen Bruch mit dem bisherigen acquis europäischer Mittelmeerpolitik beziehungsweise gegen den Aufbau einer - gegebenenfalls konkurrierenden - Parallelstruktur, von der die nördlicheren Mitgliedstaaten der Europäischen Union ausgeschlossen bleiben sollten. Immerhin hätten diese über die engen Verknüpfungen innerhalb des Unionsrahmens auch ein fundamentales Interesse an der Ausgestaltung und Vertiefung europäischer Mittelmeerpolitik, zumal Frankreich in seiner ursprünglichen Vision einer Mittelmeerunion die Finanzierung dieser über den Haushalt der Europäischen Union vorsah. ${ }^{12}$ Unausgesprochen blieb bei dem französischen Vorstoß zudem, dass Paris versuchte, sich über seine Initiative und den Ausschluss gewichtiger Mitgliedstaaten die Meinungsführerschaft in der Mittelmeerpolitik zu sichern. Im Aushandlungsprozess innerhalb der Union zeigte sich schließlich, dass der französische Vorschlag zwar inhaltlich die Idee zu einer Depolitisierung formulierte, nicht aber als depolitisierend

9 Das Schlussdokument des Gipfels von Barcelona spricht zwar von gemeinsamen Herausforderungen und Zielen, nicht aber ausdrücklich von gleichberechtigtem Miteigentum am anlässlich des Gipfels initiierten Barcelona-Prozess, vgl. Barcelona declaration adopted at the Euro-Mediterranean Conference, 27-28 November 1995.

10 Vgl. Dorothée Schmid: Die Mittelmeerunion - ein neuer französischer Motor für die europäische MittelmeerPolitik?, Deutsche Gesellschaft für Auswärtige Politik: DGAPanalyse Frankreich 1/2008, Berlin 2008, S. 5-6.

11 Vgl. Annette Jünemann: Zwei Schritte vor, einer zurück: Die Entwicklung der europäischen Mittelmeerpolitik von den ersten Assoziationsabkommen bis zur Gründung einer ,Union für das Mittelmeer', in: Rudolf Hrbek/ Hartmut Marhold (Hrsg.): Der Mittelmeerraum als Region, Europäisches Zentrum für Föderalismus-Forschung Tübingen: EZFF Occasional Papers Nr. 35, Tübingen 2008, S. 26-59, hier S. 49-50. Der seitens Frankreichs propagierte Pragmatismus in der Mittelmeerpolitik schreibt sich ein in den größeren Kontext der Konzeption französischer Außen- und besonders Europapolitik unter Sarkozy. Vgl. Claire Demesmay/Andreas Marchetti: Frankreich ist Frankreich ist Europa. Französische Europa-Politik zwischen Pragmatismus und Tradition, Deutsche Gesellschaft für Auswärtige Politik: DGAPanalyse Frankreich 1/2010, Berlin 2010.

12 Vgl. in diesem Sinne auch den Beitrag von Günter Gloser: Neue Dynamik für konkrete Ergebnisse - Barcelona-Prozess: Union für den Mittelmeerraum, in: integration 4/2008, S. 399-402. 
im Unionsgefüge selbst wahrgenommen wurde. Immerhin ging es erneut um die Frage nach einem geografischen Ausgleich, ${ }^{13}$ um dem ,zunehmenden Gewicht Deutschlands in Mittelund Osteuropa etwas entgegenzusetzen“. ${ }^{14}$ Demgemäß führte der französische Vorstoß zu starken politischen Auseinandersetzungen und belastete zwischenzeitlich den deutsch-französischen Bilateralismus. Doch auch mediterrane Mitgliedstaaten wie Italien oder Spanien zeigten sich zunächst sehr reserviert gegenüber den französischen Ideen.

Aufgrund besonders spanischer Vorbehalte und deutscher Einwände mussten die französischen Vorstellungen schlussendlich kompatibel mit der EMP ausgestaltet werden. ${ }^{15}$ Dies spiegelt sich bereits in der Genese der Bezeichnung des neuen Politikansatzes wider: Strebte Frankreich ursprünglich die Schaffung einer Mittelmeerunion an, wählte der UfM-Gründungsgipfel noch die umständliche Bezeichnung ,Barcelona-Prozess: Union für das Mittelmeer', um die Konsistenz und Kontinuität des multilateralen Beziehungsgeflechts europäischer Mittelmeerpolitik zu unterstreichen. Auf dem Folgetreffen der UfM-Außenminister im November 2008 wurde schließlich auf einen namensgebenden Verweis auf die EMP (Barcelona-Prozess) verzichtet, der aber inhaltlich im Schlussdokument bestehen blieb. ${ }^{16}$ Während mit den damit auch verbundenen inhaltlichen Kompromissen die hohe Politisierung innerhalb der Union vom Frühjahr 2008 reduziert werden konnte, geschah dies zu einem nicht geringen Preis: Entgegen dem Anspruch einer gleichberechtigten Partnerschaft erfolgte die Aushandlung und -formulierung erneut im ,Norden', während den Südanrainern wiederum kaum mehr blieb, als dem Vorschlag der UfM zuzustimmen oder diesen abzulehnen, wie von libyscher Seite praktiziert. ${ }^{17}$

Ungeachtet der oberflächlich gesehen geringeren politischen denn pragmatischen Ambition der UfM, gestaltete Paris den UfM-Gründungsgipfel am 13. Juli 2008 selbst als Treffen mit besonderer politischer Strahlkraft: Entsprechend der Bestimmung Ägyptens als südlicher Co-Präsidentschaft saßen am Vorabend des französischen Nationalfeiertags neben dem französischen Staatspräsidenten Sarkozy, als Initiator der UfM gleichzeitig deren nördlicher Co-Präsident, und seinem ägyptischen Pendant Hosni Mubarak der Präsident der Europäischen Kommission José Manuel Barroso und der Generalsekretär der Vereinten Nationen Ban Ki-moon dem Gipfel vor. Seit diesem offiziellen Start der UfM und damit der französisch-ägyptischen Co-Präsidentschaft werden französische Stellen nicht müde, die, dienende Rolle' Frankreichs im Prozess zu betonen, und versuchen, die eigenen Ambitionen herunterzuspielen. Indes besteht ungeachtet aller notwendigen Kompromissbereitschaft das Bestreben fort, Frankreich die Meinungs- und Politikführerschaft in der Mittelmeerpolitik zu sichern. Bereits institutionell zeigt sich die französische Ambition offenkundig, da mit der UfM nicht nur das bereits mit der Europäischen Nachbarschaftspolitik beschäftigte Außenministerium am Quai d'Orsay betraut ist, vielmehr wird das ,Präsidentenprojekt ' UfM auch

13 Vgl. Jean-Pierre Chevènement: France - Allemagne: Parlons franc, Paris 1996, S. 49.

14 Joachim Schild/Martin Koopmann: Der ,Sarkozy-Moment " - politische Führung in der EU am Beispiel der französischen Ratspräsidentschaft, in: integration 3/2009, S. 266-281, hier S. 276.

15 Vgl. Presse- und Informationsamt der Bundesregierung: Pressestatements von Bundeskanzlerin Merkel und Staatspräsident Sarkozy. Pressekonferenz im Gästehaus der Niedersächsischen Landesregierung, Hannover, 03.03.2008; Europäische Kommission: Mitteilung der Kommission an das Europäische Parlament und den Rat. Barcelona-Prozess: Union für den Mittelmeerraum, KOM (2008) 319; Schmid: Die Mittelmeerunion, 2008, S. 8.

16 Vgl. Council of the European Union: Barcelona Process: Union for the Mediterranean. Ministerial Conference. 3-4 November 2008. Final Declaration, Dok. 15187/08 (Presse 314). Von dieser Zusammenführung ausgeklammert bleibt die dem Prinzip des differenzierten Bilateralismus folgende Europäische Nachbarschaftspolitik, die weiterhin komplementär die Mittelmeerpolitik der Europäischen Union ergänzt. Vgl. Joint Declaration of the Paris Summit, 2008, S. 13.

17 Vgl. Jünemann: Zwei Schritte vor, einer zurück, 2008, S. 50. 
von einer Arbeitseinheit im Élysée betreut. ${ }^{18}$ Der in der französischen Außenpolitikformulierung ohnehin relativ schwache Quai d'Orsay wird unter Sarkozy noch mehr als ohnehin üblich zurückgedrängt und bleibt damit auch in Fragen der UfM marginalisiert. Durch die politisch motivierte Ansiedlung der Arbeitseinheit beim Staatspräsidenten besteht somit bereits innerhalb der institutionellen Außenpolitikformulierung in Frankreich ein gewisser Widerspruch zur inhaltlich angestrebten Depolitisierung der Mittelmeerpolitik, welche eher ein Pendant im Zentrum französischer Diplomatie im Quai d'Orsay gefunden hätte.

\section{Ägypten zwischen Mitführung und Obstruktion}

Unmittelbar nach Lancierung der Idee einer Erneuerung euro-mediterraner Beziehungen war die Reaktion der ägyptischen Regierung kritisch bis ablehnend. ${ }^{19}$ Erst mehrere Treffen von Regierungsvertretern und direkte Gespräche der Staatsoberhäupter Sarkozy und Mubarak brachten zum Jahreswechsel 2007/2008 die Wende. ${ }^{20}$ Zweifelsohne war das wiederholte Versprechen Sarkozys, eine Co-Präsidentschaft Ägyptens zu unterstützen, eine wichtige Voraussetzung für die Unterstützung der ägyptischen Regierung, konnte sie darüber doch ihre eigene Wahrnehmung als zentraler politischer, ökonomischer und kultureller Leitakteur in der Region bestätigt sehen. Ägypten sah darin auch eine logische Konsequenz seiner de facto seit 1995 übernommenen, informellen Koordinierungsrolle der arabischen MDL im Rahmen des Barcelona-Prozesses, obgleich diese von der Europäischen Union weitgehend anerkannte Rolle seitens der verbleibenden arabischen MDL (allen voran Algerien) zunehmend infrage gestellt wurde. ${ }^{21}$

Der Schritt hin zu einer Institutionalisierung euro-mediterraner Beziehungen mit der Errichtung einer Co-Präsidentschaft implizierte schon in der ersten Präsidentschaftsperiode trotz Einrichtung eines ständigen Sekretariats eine verstärkte Intergouvernementalisierung der UfM. Blieben in den Gründungsjahren der EMP einige arabische MDL aus Protest gegen israelische Politik oftmals Ministertreffen fern (zum Beispiel Außenministertreffen in Marseille 2000), ermöglichte die Co-Präsidentschaft nun eine dezidiert aktivere Politik, was im vorliegenden Fall zu einer temporären Suspendierung der UfM-Foren seitens Ägyptens führte. Diese richtete sich gegen Israels Militäroperation ,Gegossenes Blei“ im Gazastreifen 2008/2009 und die Ernennung des umstrittenen, rechtsnationalen Avigdor Lieberman zum israelischen Außenminister im März 2009. Erst in der ersten Jahreshälfte 2010 kam wieder Bewegung in das mediterrane Unionsgefüge mit der Einigung auf den Jordanier Ahmad Masa'deh als ersten Generalsekretär der UfM und dem Kompromiss der Außenminister aus Spanien, Frankreich und Ägypten, das für Juni 2010 geplante Gipfeltreffen der 43 Staatsund Regierungschefs in die zweite Jahreshälfte 2010 zu verschieben. ${ }^{22}$ Doch auch die zweite

18 Der entsprechende Zugriff des Élysée wurde bereits zur Zeit der Aushandlung der UfM gesichert. Vgl. Daniela Schwarzer: Deutschland, Frankreich und die Mittelmeerunion: Gründe für den Streit und Perspektiven für gemeinsames Handeln, in: Rudolf Hrbek/Hartmut Marhold (Hrsg.): Der Mittelmeerraum als Region, Europäisches Zentrum für Föderalismus-Forschung Tübingen: EZFF Occasional Papers Nr. 35, Tübingen 2008, S. 73-92, hier S. 85.

19 Doaa El-Bey: Tying one knot, tightening another, in: al-Ahram weekly, 02.-08.08.2007.

20 Thomas Demmelhuber: Gegenwind für die Mittelmeerunion, Konrad-Adenauer-Stiftung: KAS-Länderberichte, März 2008.

21 Vgl. dazu der Streit, welches arabische MDL die Co-Präsidentschaft von Ägypten 2010 übernehmen dürfe, in der in London herausgegebenen, arabischen Tageszeitung al-Quds al- A Arabi vom 17. Dezember 2009. Vgl. zur Führungsrolle Ägyptens und der kompromisslosen Unterstützung dieser durch die Europäische Union: Ahmed Sabry: Pushing Forward Gently, in: al-Ahram weekly, 25.06.-01.07.2009.

22 Vgl. Spanische Ratspräsidentschaft: The Union for the Mediterranean Summit, postponed to ensure its "complete success", Pressemitteilung, 24.05.2010; vgl. dazu auch: The Daily News Egypt: EU, Mediterranean Project Stalled Again: French FM, 27.10.2009. 
Jahreshälfte 2010 führte zu keinem Gipfeltreffen, sodass man sich auf das Jahr 2011 verständigte und wiederum auf die festgefahrene Konfliktkonstellation im arabisch-israelischen Konflikt als Grund für die Verschiebung verwies. Nach den noch ergebnisoffenen Umwälzungen in Ägypten und der Region ist davon auszugehen, dass vor 2012 keine neuen Impulse für die UfM zu erwarten sind, zumal sich in Ägypten die zukünftige politische Akteurskonstellation gerade erst herausbildet und das Militär nur interimsmäßig die präsidialen Machtkompetenzen übernommen hat. Aus Sicht des höchsten Militärrats in Ägypten ist das Ziel, die UfM-Institutionen noch stärker zu operationalisieren, sicherlich nur von sehr sekundärer Bedeutung, in Anbetracht sehr viel dringlicherer politischer und ökonomischer Grundsatzentscheidungen. Der inzwischen verlautbarte Verzicht Ägyptens auf die Co-Präsidentschaft eröffnet zwar die Möglichkeit zu einem geordneten Übergang in der UfM, dessen reibungsloser Ablauf aber noch lange nicht gewährleistet ist. ${ }^{23}$

Die zentrale Koordinierungsrolle Ägyptens leistete insgesamt einer eigentlich nicht intendierten Politisierung der UfM weiter Vorschub. Konnten höhere französische Beamte im Sommer 2010 noch versichern, dass die Vertagung des Gipfels durchaus positiv zu bewerten sei, da zu einem späteren Zeitpunkt das UfM-Sekretariat bereits arbeitsfähig sei und somit für die praktische Transmission der Gipfelergebnisse Sorge tragen könne, kann der zweiten Vertagung eine derart zweckoptimistische Interpretation nicht mehr abgewonnen werden. Vielmehr wird klar, wie sehr die MDL ihre gleichberechtigte Rolle in der Mittelmeerpolitik nutzen, um - entgegen der ursprünglichen Erwartungen - Politikprozesse zu blockieren. Mit den politischen Umwälzungen, die in Ägypten zum Rücktritt des Präsidenten Hosni Mubarak nach fast 30 Jahren im Amt führten, wird der Prozess, aufgrund seiner Anbindung an die Regierungen der MDL, die sich nunmehr intern herausgefordert sehen oder bereits im Wandlungsprozess sind, nicht leicht weiterzuführen sein. Die Grundannahme der UfM einer möglichen und förderlichen Depolitisierung bleibt bei einem weiterhin an politische Institutionen (Regierungen) angebundenen Prozess fraglich. Schließlich folgen die jeweiligen Partner entweder weiterhin einem eher autoritären Herrschaftsmodell oder werden möglicherweise alsbald von neuen politischen Kräften geprägt, denen die Europäische Union aber lange Zeit aufgrund ihrer Stabilitätspräferenzen die ,kalte Schulter` zeigte. Inwieweit hier also Pragmatismus im Sinne von , business as usual ' vorhalten wird, ist in der derzeitigen Interimsphase nicht zu beantworten, zumal der politische Wandlungsprozess mitsamt seinen heterogenen Akteuren ein ergebnisoffener Prozess ist und keinen offenkundigen Pfadabhängigkeiten folgt.

\section{Politische Wirklichkeit der UfM}

Auch wenn die normativen Ansprüche des Barcelona-Prozesses offiziell beibehalten wurden, ${ }^{24}$ zielte das Gründungsdokument der UfM im ursprünglichen französischen Sinne vor allem darauf ab, präzise Felder der Kooperation zu identifizieren und somit die Zusammenarbeit pragmatisch zu intensivieren. Im Wesentlichen einigten sich die Staats- und Regierungschefs in Paris auf sechs Programmlinien: erstens, die Umweltverschmutzung des mediterranen Beckens als eine grenzüberschreitende Herausforderung wahrzunehmen und demzufolge gemeinsam dagegen zu handeln; zweitens, die infrastrukturelle Verknüpfung der MDL durch transnationale Infrastrukturprojekte zu verbessern, um vor allem den SüdSüd-Handel zu erleichtern und nichttarifäre Handelshemmnisse zu verringern; drittens, die

23 Vgl. Ministère des Affaires étrangères et européennes: Intervention d'Alain Juppé lors du débat à l'Assemblée nationale sur le thème: „Europe et Méditerranée“, Paris, 30.03.2011.

24 Vgl. Joint Declaration of the Paris Summit, 2008, S. 12. 
Zusammenarbeit im Zivil- und Katastrophenschutz zu verbessern; viertens, die Kooperation in der Erschließung der Region für die Nutzung alternativer Energien zu intensivieren (vor allem Sonnenenergie); fünftens, das Beziehungsgeflecht im studentischen und akademischen Austausch zu verdichten (durch die Programme Erasmus und Tempus) und mit einer euro-mediterranen Universität (EMUNI University) zu institutionalisieren; und schließlich sechstens, die ökonomischen Rahmenbedingungen, das heißt vor allem das Investitionsklima in enger, noch weiter zu vertiefender Zusammenarbeit mit dem Privatsektor konkurrenzfähig zu machen. ${ }^{25}$ Mit Blick auf die politische Transformation weiter Teile des Mittelmeerraums ist für die Gegenwart und Zukunft der UfM zu hinterfragen, ob die benannten und in depolitisierender Absicht identifizierten Themen und Projekte auch aus Sicht der MDL tragfähige Impulse der Kooperation bleiben werden.

\section{Institutionalisierung zwischen Politik- und Projektausrichtung}

Zur Verwirklichung der genannten Agenda folgt die politische Grundstruktur der UfM der Logik der bereits in Barcelona angestoßenen Verstetigung der euro-mediterranen Beziehungen durch Festlegung regelmäßiger Treffen und gemeinsamer Foren. Das Schlussdokument von Paris sieht alle zwei Jahre die Abhaltung eines euro-mediterranen Gipfels vor, der durch jährliche Außenministertreffen ergänzt wird. ${ }^{26}$ Hochrangige Beamte (, senior officials`) der 43 UfM-Teilnehmerstaaten sollen sich regelmäßig treffen, unter anderem die Ministertreffen vorbereiten und den UfM-Außenministern das jährliche Arbeitsprogramm der UfM vorlegen. Dabei werden sie unterstützt von einem in Brüssel angesiedelten gemeinsamen ständigen Ausschuss (, Joint Permanent Committee ') ${ }^{27}$ Anlässlich der euro-mediterranen Außenministertagung im November 2008 in Marseille, der die Ausarbeitung der Details oblag, wurden die Aufgaben und Zuständigkeiten des gemeinsamen ständigen Ausschusses und der höheren Beamten präzisiert sowie die bisherigen Aufgaben des Euromed-Ausschusses auf diese übertragen und folglich letztgenannter aufgelöst. ${ }^{28}$ Entscheidungen innerhalb der Gremien der UfM werden - ungeachtet der Öffnung für Projekte entsprechend dem Prinzip der variablen Geometrie - im Konsens getroffen. ${ }^{29}$ Aufgrund der gleichberechtigten Beteiligung aller 43 Teilnehmerstaaten, geht damit eine Intergouvernementalisierung im Vergleich zur bisherigen Mittelmeerpolitik der Europäischen Union einher, die sich nun nicht mehr über die ,wechselseitigen Proteste und Verweigerungen [einzelner MDL] hinwegsetzen" kann. ${ }^{30}$ Mit der häufig eingeforderten Aufgabe der formalen Asymmetrie in den NordSüd-Beziehungen verzichtet die Europäische Union damit auf die Möglichkeit, den Politikprozess auch gegen Widerstände der MDL voranzutreiben, wodurch sie aber nolens volens einer möglichen Politisierung seitens der MDL Tür und Tor geöffnet hat.

Im Sinne der gemeinsamen Verantwortung für den Politikprozess muss die Einrichtung der auf ,Nord ' und ,Süd ' verteilten Co-Präsidentschaft zunächst als eine der zentralen Innovationen der UfM gegenüber der EMP gesehen werden. Gemeinsam stehen die beiden Co-Prä-

25 Vgl. ebenda, S. 19-20.

26 Vgl. ebenda, S. 13.

27 Vgl. ebenda, S. 15-16.

28 Vgl. Council of the European Union: Barcelona Process: Union for the Mediterranean, 2008, S. 7-8. Eine Übersicht zur Genese der einzelnen Gremien von der Euro-Mediterranen Partnerschaft zur Union für das Mittelmeer entsprechend der Pariser Erklärung bieten Roberto Aliboni/Fouad M. Ammor: Under the Shadow of ,Barcelona': From the EMP to the Union for the Mediterranean, Euro-Mediterranean Study Commission: EuroMeSCo Paper 77, 2009, S. 29-30.

29 Joint Declaration of the Paris Summit, 2008, S. 11 und 16.

30 Gerrit F. Schlomach: Union für das Mittelmeer - Realitäten anerkennen und Chancen nutzen!, in: KAS-Auslandsinformationen 9/2010, S. 117-134, hier S. 131. 
sidenten den Tagungen der UfM auf allen Ebenen vor. Allerdings ist die Co-Präsidentschaft, wie bereits beispielhaft an den die Gründungs-Co-Präsidentschaft tragenden Staaten Frankreich und Ägypten aufgezeigt, keinesfalls frei von über den propagierten Partnerschaftsgedanken hinausgehenden politischen Erwägungen, Zwängen und Partikularinteressen. Zudem ist mit Blick auf die bisherigen Erfahrungen aber auch auf kommende Präsidentschaften - bereits die konkrete Besetzung und Ausgestaltung der Co-Präsidentschaft beiderseits des Mittelmeers weit weniger evident, als auf Basis der UfM-Gründungsdokumente anzunehmen wäre.

Die Pariser und Marseiller Erklärungen besagen für den ,Südvorsitz', dass hierfür ein MDL für die Dauer von zwei Jahren im Konsens bestimmt wird, dessen Mandat nicht erneuerbar ist. ${ }^{31}$ Entsprechend der vorgesehenen Abfolge der Gipfelbegegnungen sollte die Benennung der ,Süd-Präsidentschaft' zeitlich mit dem alle zwei Jahre stattfindenden euro-mediterranen Gipfel wechseln. Nahezu klassisch stellen sich aufgrund der Heterogenität der MDL die künftigen Konfliktlinien zur Bestimmung dieser Präsidentschaft dar, beispielsweise wären nicht viele arabische MDL als Co-Präsidenten für Israel akzeptabel, umgekehrt wäre aber auch Israel für alle arabischen Staaten inakzeptabel. Zwischen diesen könnte es aber auch zu vermehrter Uneinigkeit kommen, sofern sowohl politische Transformation als auch autoritäre Persistenz die mittelfristige Realität der arabischen MDL bestimmen. Ebenfalls als nur schwer durchsetzbar könnten sich Ambitionen der Balkan-MDL oder der Türkei zur Übernahme der ,Süd-Präsidentschaft' erweisen, da die arabischen Mittelmeeranrainer als weiterhin bedeutendste Gruppe der MDL wenig geneigt sein dürften, eine nicht-arabische ,Süd-Präsidentschaft‘ zu billigen.

Für den ,Nordvorsitz' legen die Abschlussdokumente von Paris und Marseille fest, dass die Präsidentschaft seitens eines Mitgliedstaates der Europäischen Union erfolgt. Es wurde lapidar - aber mit umso größeren politischen Implikationen - hinzugefügt, dass diese Präsidentschaft mit der in den Verträgen festgelegten Außenvertretung der Europäischen Union kompatibel sein müsse. ${ }^{32}$ Damit gerät die Frage nach der Besetzung der, Nord-Präsidentschaft ${ }^{\star}$ in das Fahrwasser der politischen Aushandlungsprozesse um die konkrete Ausgestaltung der diesbezüglichen Bestimmungen des seit 1. Dezember 2009 geltenden Vertrags von Lissabon. Dieser sieht die Außenvertretung der Union beim hauptamtlichen Präsidenten des Europäischen Rates, unbeschadet der Kompetenzen des Hohen Vertreters der Union für Außen- und Sicherheitspolitik (Art. 15 Abs. 6 Vertrag über die Europäische Union), der gleichzeitig Vizepräsident der Kommission ist. ${ }^{33}$ Entsprechend der Unschärfe der geltenden Bestimmungen und angesichts der Tatsache, dass bis dato die Außenvertretung dem jeweiligen Ratsvorsitz oblag (Art. 18 Abs. 1 Vertrag über die Europäische Union in der Fassung des Vertrags von Nizza) $)^{34}$ - ergab sich bereits mit der Beendigung der französischen Ratspräsidentschaft Ende 2008 die Frage, inwieweit die ,Nord-Präsidentschaft ' in der UfM nicht von Frankreich auf die halbjährlich wechselnde Ratspräsidentschaft in der Europäischen Union übergehen solle. Zwar konnte Frankreich seinen Führungsanspruch bisher wahren ${ }^{35}$ und auch gegenüber Spa-

31 Vgl. Joint Declaration of the Paris Summit, 2008, S. 15; Council of the European Union: Barcelona Process: Union for the Mediterranean, 2008, S. 6.

32 Vgl. Joint Declaration of the Paris Summit, 2008, S. 15; ebenso Council of the European Union: Barcelona Process: Union for the Mediterranean, 2008, S. 6, worin in diesem Kontext zudem die Rolle der Europäischen Kommission hervorgehoben wird.

33 In ihrer Stellungnahme vom Mai 2008 sah die Kommission mit Verweis auf den - damals noch nicht in Kraft gesetzten - Vertrag von Lissabon den Vorsitz auf Ebene der Staats- und Regierungschefs beim Präsidenten des Europäischen Rats und dem Kommissionspräsidenten, auf Ebene der Außenminister beim Hohen Vertreter. Vgl. Europäische Kommission: Barcelona-Prozess: Union für den Mittelmeerraum, 2008, S. 7.

34 Vgl. ebenda.

35 So zum Beispiel vermittels der französischen Arrangements mit dem tschechischen und schwedischen Ratsvorsitz. Vgl. Barbara Lippert: Europäische Nachbarschaftspolitik, in: Werner Weidenfeld/Wolfgang Wessels (Hrsg.): Jahrbuch der Europäischen Integration 2009, Baden-Baden 2010, S. 229-240, hier S. 233. 
nien aufrechterhalten, das selbst ,mediterran" ambitioniert ist. Klarheit ist jedoch bisher nicht gegeben, auch wenn Frankreich weiterhin die nördliche Co-Präsidentschaft zugesprochen wird, so beispielsweise auch seitens des für die Erweiterungs- und Nachbarschaftspolitik zuständigen Kommissionsmitglieds Štefan Füle. ${ }^{36}$ Auch der infolge des Vertrags von Lissabon eingerichtete Europäische Auswärtige Dienst dürfte mittelfristig hieran nichts ändern, schließlich sehen die Gründungsdokumente der UfM die Co-Präsidentschaft von staatlicher und nicht von institutioneller Seite gewährleistet.

Neben den politischen Implikationen und verfahrenstechnischen Schwierigkeiten, die Co-Präsidentschaften zu benennen, könnte - aufgrund der den MDL anzulastenden wiederholten Vertagung des Pariser Nachfolgegipfels einerseits und den Unklarheiten bezüglich der außenpolitischen Vertretung der Europäischen Union und damit der Bestimmung des europäischen Co-Präsidenten andererseits - künftig das Problem eines nicht gewollten zeitlichen Auseinanderklaffens in der ,nördlichen “ und ,südlichen ‘ Besetzung der Co-Präsidentschaft auftreten, ${ }^{37}$ was der Kohärenz und Zurechenbarkeit der UfM-Prozesse weiter abträglich wäre.

\section{Das Sekretariat der Union für das Mittelmeer}

Ist die Co-Präsidentschaft per se durchaus als politische Einrichtung zu begreifen, ist in der Errichtung eines gemeinsamen Sekretariats als die zweite zentrale Innovation der UfM zunächst ein depolitisierender Antrieb zu sehen. Entsprechend betonen die Abschlussdokumente von Paris und Marseille gleichermaßen die technische Natur des Sekretariats und sprechen ihm, mit Verweis auf die Kompetenzen der Außenminister und hochrangigen Beamten, eine politische Rolle ab. ${ }^{38}$ Das Sekretariat soll im Rahmen der UfM eine zuarbeitende, koordinierende und gegebenenfalls auch implementierende Rolle spielen. ${ }^{39}$ Dessen ungeachtet sind auch mit Blick auf dieses technische Sekretariat in mehrfacher Hinsicht Tendenzen einer nicht intendierten Politisierung auszumachen.

Zunächst konnte sich Frankreich mit seiner Unterstützung für eine Ansiedlung des Sekretariats in Tunis nicht durchsetzen. ${ }^{40}$ Vielmehr fiel die endgültige Wahl auf Barcelona, das sich zunehmend als Zentrum euro-mediterraner Aktivitäten etablieren kann. Immerhin ist vor Ort neben dem UfM-Sekretariat mit dem European Institute of the Mediterranean (IEMed) ein in euro-mediterranen Fragen relevantes Forschungsinstitut angesiedelt, dem es zudem gelang, 2010 die Koordination des euro-mediterranen Kooperationsnetzwerks EuroMeSCo (Euro-Mediterranean Study Commission) zu übernehmen. Welche Implikationen diese Verdichtung in Barcelona auf die Aushandlung des französischen und spanischen Führungsanspruchs in mediterranen Fragen haben wird, bleibt abzuwarten. Eine Verringerung politischer Einflussversuche dürfte hieraus aber nicht abzuleiten sein.

36 Štefan Füle: Address to the "For'UM" meeting, 27.05.2010, SPEECH/10/269. Vgl. aus französischer Sicht auch Ministère des Affaires étrangères et européennes: Bilan et avenir de l'union pour la méditérranée. Discussion d'une question orale avec débat au Sénat. Intervention de Mme Michèle Alliot-Marie, ministre d'État, ministre des affaires étrangères et européennes, Paris, 16.02.2011.

37 Vgl. Aliboni/Ammor: Under the Shadow of ,Barcelona', 2009, S. 6.

38 Vgl. Joint Declaration of the Paris Summit, 2008, S. 15; Council of the European Union: Barcelona Process: Union for the Mediterranean, 2008, S. 8.

39 Vgl. Council of the European Union: Barcelona Process: Union for the Mediterranean, 2008, S. 8-9; sowie die im März 2010 - um dreizehn (!) Monate verspätet - verabschiedeten Statuten des Sekretariats: Statutes of the Secretariat of the Union for the Mediterranean, 2010, S. 2 und 6.

40 Vgl. Ivan Camilleri: Future of Med. Union in doubt. All scheduled meetings postponed due to Gaza war, in: Times of Malta, 09.01.2009. 
Aufgrund politisch bedingter Verzögerungen erfolgte die Einrichtung des Sekretariats erst im März 2010, als schließlich der Jordanier Masa'deh offiziell als Generalsekretär eingeführt werden konnte. Doch auch in der Benennung der stellvertretenden Generalsekretäre offenbarte sich erneut das Dilemma einer unmittelbaren Politisierung des Sekretariats bei gleichzeitiger Depolitisierung seiner Rolle. Obwohl der Arabischen Liga bereits anlässlich des Gipfels von Paris Beobachterstatus in der UfM zugesprochen worden war, ${ }^{41}$ bestand Israel als Gegenleistung für deren praktische Akzeptanz auf einem eigenen stellvertretenden Generalsekretär. ${ }^{42}$ Im Sinne einer ausgleichenden Besetzung ging hiermit eine als inflationär einzuschätzende Postenpolitik einher, sodass sich die UfM-Außenminister zunächst darauf verständigten, dem Generalsekretär insgesamt fünf Stellvertreter an die Seite zu stellen. ${ }^{43}$ In den Statuten des Sekretariats wird diese Zahl sogar auf sechs erhöht, ${ }^{44}$ sodass neben den genannten auch ein Stellvertreterposten seitens der Türkei besetzt wird (siehe Abbildung 1).

Aufgrund dieser Regelungen können sich in der Besetzung des Sekretariats politisch gesehen die meisten staatlichen UfM-Akteure - zumindest indirekt - repräsentiert sehen. Damit einher geht aber auch die Gefahr einer Blockade des Sekretariats, sollten sich politische Konflikte trotz der Zuordnung thematischer Portfolios zu den einzelnen stellvertretenden Generalsekretären - ganz im Geiste des pragmatischen Ansatzes einer deutlicheren Projektorientierung - in seiner Arbeit fortsetzen. Nach französischer Lesart soll mit der gegebenen Besetzung gerade eine Blockade des Sekretariats verhindert werden, immerhin seien auch jene an Bord und damit in der Verantwortung, die sich politisch als ,problematisch "erweisen könnten. Inwieweit diese Rechnung aufgeht und die Unabhängigkeit des Sekretariats gewahrt bleibt, wird sich erst noch zeigen müssen. Wenig verwunderlich ist angesichts der politischen Erwägungen und den daraus notwendig werdenden Kompromissen, dass sich weder Frankreich noch Ägypten herausragende Posten im Sekretariat sicherten, ebenfalls wie das bereits den Sitz des Sekretariats stellende Spanien. Dieses fragile Gleichgewicht ist mit dem (überraschenden) Rücktritt Masa'dehs vom Amt des Generalsekretärs während der Phase der Einrichtung des Sekretariats empfindlich gestört worden. Sein Rückzug, offiziell aufgrund veränderter Ausgangsbedingungen innerhalb der UfM (vor allem finanzieller Natur) im Vergleich zu jenen im Jahr 2010, wurde zunächst durch die Ernennung des ersten stellvertretenden Generalsekretärs Lino Cardarelli aus Italien zu seinem Nachfolger auf Interimsbasis zum 1. März 2011 aufgefangen. Indes ist absehbar, dass die endgültige Besetzung des Sekretariats weiterhin politischen Zündstoff bietet.

Verwunderlich ist angesichts der genannten institutionellen Austarierung die derzeit klar mediterrane Ausrichtung in der Postenvergabe des Sekretariats: Entgegen des Frankreich abgerungenen Zugeständnisses, die ursprünglich geplante Mittelmeerunion mit der UfM als Fortsetzung der EMP unter Einbezug aller Mitgliedstaaten der Europäischen Union auszugestalten, ist seitens der Mitgliedstaaten im Sekretariat kein stellvertretender Generalsekretär aus einem Nicht-Mittelmeeranrainerstaat berücksichtigt - lediglich bei deren Mitarbeitern sind auch Nicht-Anrainer anzutreffen. Entsprechend scheint auf Seiten der Europäischen Union eine Führungsrolle der Mittelmeeranrainer in der UfM bis auf Weiteres gegeben, auch wenn diese Frankreich trotz aller Ambitionen nicht mit absoluter Gewissheit zugeordnet werden kann, auch aufgrund der Tatsache, dass diese Rolle nicht unangefochten ist.

41 Vgl. Joint Declaration of the Paris Summit, 2008, S. 8.

42 Vgl. Schlomach: Union für das Mittelmeer, 2010, S. 126.

43 Vgl. Council of the European Union: Barcelona Process: Union for the Mediterranean, 2008, S. 9.

44 Vgl. Statutes of the Secretariat of the Union for the Mediterranean, 2010, S. 2. 
Abbildung 1: Struktur des (,ersten`) UfM-Sekretariats zum 28. Februar 2011

\begin{tabular}{|l|l|l|l|l|l|}
\hline $\begin{array}{l}\text { Generalsekretär: Ahmad Masa'deh (Jordanien) } \\
+5 \text { Mitarbeiter }\end{array}$ \\
$\begin{array}{l}\text { Projektförderung } \\
\text { und Unterneh- } \\
\text { mensentwicklung }\end{array}$ & $\begin{array}{l}\text { Soziale An- } \\
\text { gelegenheiten }\end{array}$ & $\begin{array}{l}\text { Transport und } \\
\text { Stadtent- } \\
\text { wicklung }\end{array}$ & $\begin{array}{l}\text { Bildung } \\
\text { und } \\
\text { Forschung }\end{array}$ & $\begin{array}{l}\text { Umwelt und } \\
\text { Wasser }\end{array}$ & Energie \\
\hline $\begin{array}{l}\text { erster stellv. } \\
\text { Generalsekretär }\end{array}$ & $\begin{array}{l}\text { stellv. } \\
\text { Generalsekretär }\end{array}$ & $\begin{array}{l}\text { stellv. } \\
\text { Generalsekretär }\end{array}$ & $\begin{array}{l}\text { stellv. } \\
\text { General- } \\
\text { sekretär }\end{array}$ & $\begin{array}{l}\text { stellv. } \\
\text { Generalsekretär }\end{array}$ & $\begin{array}{l}\text { Gtellv. } \\
\text { Generalsekretär }\end{array}$ \\
\hline $\begin{array}{l}\text { Lino Cardarelli } \\
\text { (Italien) }\end{array}$ & $\begin{array}{l}\text { Cecilia } \\
\text { Attard-Pirotta } \\
\text { (Malta) }\end{array}$ & $\begin{array}{l}\text { Yigit Alpogan } \\
\text { (Türkei) }\end{array}$ & $\begin{array}{l}\text { Ilan Chet } \\
\text { (Israel) }\end{array}$ & $\begin{array}{l}\text { Rafiq Husseini } \\
\text { (Palästinensische } \\
\text { Autonomiebehörde) }\end{array}$ & $\begin{array}{l}\text { NN } \\
\text { (Griechenland) }\end{array}$ \\
\hline + 1 Mitarbeiter & +1 Mitarbeiter & +1 Mitarbeiter & +1 Mitarbeiter & + 2 Mitarbeiter \\
\hline
\end{tabular}

Quelle: Eigene Darstellung auf der Basis von Angaben des UfM-Sekretariats.

Aus dem Gesagten folgt die Frage nach den Zuständigkeiten und Policy-Verschränkungen in der Praxis, gerade auch im Verhältnis der Europäischen Nachbarschaftspolitik zur UfM. Immerhin erfolgt die Finanzierung der UfM-Aktivitäten und des Sekretariats über das Unionsbudget - und hier insbesondere über das Europäische Nachbarschafts- und Partnerschaftsinstrument (ENPI) - sowie freiwillige Beiträge der Teilnehmerstaaten. ${ }^{45}$ Indes sind die derzeitigen Möglichkeiten einer umfassenden UfM-Finanzierung aus dem Unionsbudget eingeschränkt, weshalb die in der sechsten Programmlinie (laut Pariser Erklärung) erwähnte, weitere Öffnung für den Privatsektor in erster Linie vor dem Hintergrund eines geringen finanziellen Spielraums der Europäischen Union zu betrachten ist - trotz Umwidmung schon bereitgestellter Mittel aus dem Unionshaushalt (2007-2013) für die seit Paris 2008 konkretisierte Prioritätenliste euro-mediterraner Kooperation. ${ }^{46}$

Gegenwärtig gegebene Unklarheiten privilegieren die intergouvernementalen Entscheidungsgremien und damit eine nicht immer förderliche Politisierung. Dies ist auch ungeachtet der Einrichtung eines technischen Sekretariats zu konstatieren. Schließlich könnte sich die UfM - in ihren Entscheidungen ohnehin zwischenstaatlich angelegt - mit ihm durchaus auch vom supranationalen Zugriff der Europäischen Kommission über die unionseigenen Finanzierungsinstrumente entfernen, immerhin bestand wohl eine französische Intention gerade darin, mit dem UfM-Sekretariat in euro-mediterranen Fragen die Kommission zu schwächen. ${ }^{47}$ Auch mit Blick auf den Vertrag von Lissabon ist zwar in vielen Politikbereichen eine stärkere - partizipative - Öffnung festzustellen, gleichzeitig wurde aber im außenpolitischen Handeln die intergouvernementale Anlage europäischer Politik bestätigt. ${ }^{48}$ Für eine entsprechende Tendenz in der Mittelmeerpolitik spricht auch, dass zwar eine stärkere Parlamentarisierung in den Gründungsdokumenten der UfM angestrebt wird, ${ }^{49}$ diese bisher

45 Vgl. Joint Declaration of the Paris Summit, 2008, S. 17; Council of the European Union: Barcelona Process: Union for the Mediterranean, 2008, S. 10.

46 Vgl. Schlomach: Union für das Mittelmeer, 2010, S. 131.

47 Vgl. Aliboni/Ammor: Under the Shadow of ,Barcelona', 2009, S. 10.

48 Vgl. Franco Algieri/Thomas Bauer: Die Festschreibung mitgliedstaatlicher Macht: GASP und GSVP im Vertragswerk von Lissabon, in: Werner Weidenfeld (Hrsg.): Lissabon in der Analyse. Der Reformvertrag der Europäischen Union, Baden-Baden 2008, S. 125-156, hier S. 152.

49 Vgl. Joint Declaration of the Paris Summit, 2008, S. 14; Council of the European Union: Barcelona Process: Union for the Mediterranean, 2008, S. 10. Vgl. angesichts der Umwälzungen im südlichen Mittelmeerraum erneut Europäischer Rat: Außerordentliche Tagung des Europäischen Rates, 11. März 2011. Erklärung, EUCO 7/11, S. 1. 
aber nicht erfolgt ist und auch bei der Wahl des UfM-Generalsekretärs die Chance zur Konsultation der Parlamentarischen Versammlung Europa-Mittelmeer nicht genutzt wurde.$^{50}$

\section{Programmlinien - Design und Realität}

Entsprechend der problematischen Rahmenbedingungen fällt mehr als zwei Jahre nach Gründung und Präzisierung der UfM auch eine Bilanz in den einzelnen Kooperationsfeldern mit einigen Ausnahmen dürftig aus. Positiv zu vermerken sind auf den ersten Blick die Gründung einer euro-mediterranen Universität (EMUNI University) im slowenischen Portorož, die Initiative eines euro-mediterranen solarthermischen Verbundnetzes (Mediterranean Solar Plan, MSP) oder der Versuch, die Verschmutzung des mediterranen Beckens zu verringern. Dennoch sind das nicht genuin aus der UfM und ihrem depolitisierenden Ansatz hervorgegangene Initiativen. Vielmehr waren sie einerseits bereits vorher angedacht, wie sich am Beispiel der Universitätsgründung am 9. Juni 2008 noch während der slowenischen Ratspräsidentschaft, also einen Monat vor der offiziellen Gründung der UfM, zeigt. ${ }^{51}$ Andererseits handelt es sich bei Projektprioritäten wie im Falle der Förderung alternativer Energien im südlichen Mittelmeerraum um Themenbereiche, die in die UfM inkorporiert wurden, um den politischen Willen für von Industrie und Investoren angestoßene, ambitioniertere Projekte zu unterstreichen. So wurde das Projekt DESERTEC bereits im Jahre 2008 diskutiert und 2009 mit der Gründung der DESERTEC Foundation formalisiert. ${ }^{52}$ Sowohl der mediterrane Solarplan als auch DESERTEC streben die Nutzbarmachung von Sonnen- und Windenergie in Nordafrika und den anteiligen Export des aus alternativen Energien gewonnenen Stroms nach Europa an. ${ }^{53}$ Gleichwohl gilt auch hier, dass die von der ägyptischen Co-Präsidentschaft angestoßene Suspendierung von Ministertreffen (hier: Verschiebung des Treffens der Energieminister im Juni 2010 in Kairo) zu einer deutlichen Verschleppung der weiteren Implementierung des Solarplans führte. Ähnliches zeigt sich bei der im Gründungsdokument der UfM anvisierten Zusammenarbeit im Bereich Schutz des Mittelmeers vor Verschmutzung (erste Programmlinie). Rekurriert wird in dieser Projektlinie zuvorderst auf eine Initiative, die bereits 2006 von der Europäischen Kommission initiiert ${ }^{54}$ und von den Mitgliedern der EMP (Treffen der Umweltminister in Kairo im November 2006) auch angenommen wurde. So konnte zwar unter Rückgriff auf die sogenannten ,Horizon 2020'-Leitlinien und die Schlusserklärung von Paris eine prinzipielle Einigung auf eine gemeinsame Wasserstrategie erreicht werden. Politische Streitigkeiten, etwa die Bezugnahme auf den Terminus , besetzte Gebiete“ im Kontext des Nahostkonflikts, verhinderten dennoch im April 2010 bei einem Treffen der euro-mediterranen Fachminister für

50 Vgl. Schlomach: Union für das Mittelmeer, 2010, S. 128.

51 Das Gründungsstatut wurde dann schließlich am 26. November 2008 verabschiedet.

52 Präambel im Memorandum of Understanding der DESERTEC Industrial Initiative (DII): „Therefore, the signatories of this MoU intend to establish a planning entity in order to create a basis for putting the DESERTEC concept into practice as soon as possible. Work on the Mediterranean Solar Plan (Union for the Mediterranean) are intended to be included in this effort." DESERTEC Industrial Initiative: Memorandum of Understanding regarding the establishment of a DESERTEC Industrial Initiative planning entity (DII) based on the DII principles, 13.07.2009. Zum Verhältnis der beiden Initiativen siehe auch die Pressemitteilung der DESERTEC-Initiatoren Trans-Mediterranean Renewable Energy Cooperation (TREC): Solar-Plan wird offizielles Flaggschiff-Projekt der Union für das Mittelmeer, 05.12.2008.

53 Isabelle Werenfels/Kirsten Westphal: Solarstrom aus Nordafrika. Rahmenbedingungen und Perspektiven, Stiftung Wissenschaft und Politik: SWP-Studie S3, Berlin 2010.

54 Europäische Kommission: Mitteilung der Kommission an den Rat und das Europäische Parlament. Bestimmung einer Umweltstrategie für den Mittelmeerraum, KOM (2006) 475. 
Wasser in Barcelona eine Annahme dieser Strategie seitens der euro-mediterranen Regierungsvertreter. ${ }^{55}$

Kritisch ist ferner die im Grundsatz anvisierte, verstärkte Einbindung des Privatsektors in die Ausgestaltung euro-mediterraner Beziehungen zu hinterfragen. Am Beispiel der sechsten Programmlinie, die darauf abzielt, das Investitionsklima in der Region zu stärken und hierfür auch vermehrt die Zusammenarbeit mit privaten Investoren zu suchen, lässt sich dies anschaulich nachzeichnen. Im Zuge von ökonomischen Strukturanpassungen in den Staaten des südlichen Mittelmeerraums ist länderübergreifend eine Stärkung des Privatsektors in der Wirtschaft und in der Politik zu beobachten. Vor allem das Fallbeispiel Ägypten verdeutlicht, wie private Unternehmer während der Präsidentschaft Mubaraks aufgrund ihrer guten Zugänge zu politischen Entscheidungsträgern oligopolartige Stellungen in den jeweiligen Märkten aufbauen konnten, durch Übernahme politischer Mandate und Ämter auch Einfluss auf die tatsächliche Politikformulierung nehmen und zu einem konstitutiven Element in der Herrschaftselite geworden sind. ${ }^{56}$ Ein Beispiel hierfür ist der ehemalige ägyptische Industrie- und Außenhandelsminister Rachid Mohamed Rachid, dessen Familienunternehmen, dem er bis zu seiner Amtsübernahme 2004 auch vorstand, sich seit 30 Jahren im ägyptischen und nahöstlichen Genuss- und Nahrungsmittelsektor etabliert hat. Rachid hat 2009 im Rahmen der UfM in seiner Funktion als Außenhandelsminister die Bildung eines neuen, öffentlich-privaten Finanzierungsinstruments (InfraMed) vorangetrieben, das dem Ziel der Schlusserklärung von Paris entspricht, in den euro-mediterranen Beziehungen auch private Investoren in die Projektfinanzierung mit einzubeziehen. ${ }^{57}$ Getragen wird dieser Investitionsfonds von fünf öffentlichen beziehungsweise privaten Banken aus Frankreich (Caisse des Dépôts), Italien (Cassa Depositi e Prestiti), Marokko (Caisse de Dépôt et de Gestion), Ägypten (EFG-Hermes) und der Europäischen Union (Europäische Investitionsbank, EIB) mit einem gesicherten Mindestgründungskapital von 385 Millionen Euro (angestrebt werden eine Milliarde Euro). Rachid wurde von den Investoren in den erweiterten Vorstand von InfraMed gewählt, um offenkundig seine Unternehmerexpertise und sein politisches Gewicht in die strategische Ausrichtung des Fonds einfließen zu lassen. ${ }^{58}$ Von ägyptischer Seite wird dieser Fonds von der Investmentbank EFG-Hermes getragen, an der Rachid neben einigen seiner früheren ägyptischen Kabinettskollegen aber bis 2006 selbst Anteile im Wert von knapp 100 Millionen Euro hielt. ${ }^{59}$ In diesem Fall sind Zweifel angebracht, ob durch eine derartige Verquickung von politischen und ökonomischen Interessen noch eine unabhängige Prüfung von Projekten im südlichen Mittelmeerraum und ein Mindestmaß an Transparenz möglich sind. Diese nationalspezifische Verquickung politischer Entscheidungsträger mit Unternehmerinteressen ist hinreichend bekannt und in der Literatur als Herausforderung für Entwicklungszusammenarbeit beschrieben. ${ }^{60}$ Neu ist nun hingegen, dass sich dieses regio-

55 Vgl. dazu auch Antwort der Bundesregierung auf die Kleine Anfrage der Fraktion der SPD, - Drucksache 17/ 2524 - Zwei Jahre Union für das Mittelmeer, Bundestagsdrucksache 17/2669, S. 2; Pierre Ausseill: Med Water Conference Ends in Failure Due to Israel-Arab Row, in: The Daily News Egypt, 14.04.2010.

56 Vgl. Demmelhuber: EU-Mittelmeerpolitik und der Reformprozess in Ägypten, 2009, S. 182-209.

57 Vgl. EFG-Hermes: EFG-Hermes, Caisse des Dépôts, Cassa Depositi e Prestiti and Caisse de Dépôt et de Gestion announce the launch of InfraMed - the first long-term investment fund and the first financing facility of the Union for the Mediterranean, Pressemitteilung, 30.04.2009.

58 Vgl. Caisse des Dépôts (CDC)/Cassa Depositi e Prestiti (CDP)/the European Investment Bank (EIB)/Caisse de Dépôt et de Gestion (CDG)/EFG Hermes: As a major contribution to the Union for the Mediterranean: InfraMed Infrastructure Fund is launched with EUR 385 million at First Close The Fund volume is targeted at EUR 1 billion, 26.05.2010, BEI/10/78.

$59 \mathrm{Zu}$ den Beteiligungen des Ministers, vgl. Stephan Roll: Finance Matters! The Influence of Financial Sector Reforms on the Development of the Entrepreneurial Elite in Egypt, in: Mediterranean Politics 3/2010, S. 349-370.

60 Vgl. Thomas Demmelhuber/Stephan Roll: Herrschaftssicherung in Ägypten. Zur Rolle von Reformen und Wirtschaftsoligarchen, Stiftung Wissenschaft und Politik: SWP-Studie S20, Berlin 2007. 
nale Phänomen auch verstärkt in der euro-mediterranen Zusammenarbeit widerspiegelt, zumal dies zentralen Prämissen (Transparenz) in der mit öffentlichen Geldern geförderten, euro-mediterranen Wirtschaftszusammenarbeit widerspricht. Die umfassenden politischen Umwälzungen in Nordafrika haben nicht nur die ,Karten der politischen Macht" neu gemischt. Auch wirtschaftspolitisch finden aktuell noch ergebnisoffene Prozesse des Elitenaustauschs und der Marginalisierung einzelner Sektoreliten statt, die auch politisch motivierte Großunternehmer wie den oben genannten Rachid betreffen. Dennoch, die Verquickung von Politik und Wirtschaft ist in den Staaten Nordafrikas durch ökonomische Strukturanpassungsprogramme systemisch geworden, sodass sich daraus auch zukünftig Herausforderungen für die europäische Mittelmeerpolitik ableiten.

\section{Zwischenzeugnis der UfM und Ausblick}

Gemessen am ursprünglichen Ziel, über die UfM den euro-mediterranen Beziehungen einen neuen Schub zu verleihen, fällt das Zwischenzeugnis ernüchternd aus. Die angestrebte Depolitisierung konnte trotz inhaltlicher und institutioneller Neuakzentuierung nicht erreicht werden. Im Gegenteil führten die Betonung des Partnerschaftsgedankens und die damit einhergehende Stärkung des intergouvernementalen Charakters der Kooperation zu vermehrter Politisierung. Die von der ägyptischen Co-Präsidentschaft initiierte Suspendierung von UfM-Aktivitäten nach den israelischen Militäraktionen 2008/2009 und dem Regierungswechsel 2009 zeigte wiederholt, dass die UfM kaum eine Rückkehr auf die Projekt- und Sachebene und damit eine Depolitisierung der Zusammenarbeit erreicht. Was die Institutionalisierung europäischer Mittelmeerpolitik betrifft, bleibt zwar vor allem für das neu geschaffene Sekretariat der UfM in Barcelona die Hoffnung, dass sich dieses zu einem Motor euro-mediterraner Kooperation entwickelt und zu einer Eigendynamik in den anvisierten Kooperationsfeldern führt. Diesbezüglich ist aber von einem langwierigen Prozess auszugehen, ${ }^{61}$ nicht zuletzt wegen der ohnehin schon hohen Politisierung dieser Institution im Zuge der inflationären Postenpolitik, die dazu dient, divergierenden, politischen Partikularinteressen vor allem der MDL Rechnung zu tragen.

Die UfM fügt sich nahtlos in die Genese europäischer Mittelmeerpolitik seit 1995 ein. Die Mittelmeerpolitik der Europäischen Union stand über viele Jahre aufgrund fehlender Glaubwürdigkeit in der Kritik. Konkurrierende Politikkonzepte und eine komplexe Akteursvielfalt in der Politikformulierung waren hier vor allem verantwortlich. Die mit der Europäischen Sicherheitsstrategie von 2003 bereits eingetretene Stärkung von Stabilitäts- und Sicherheitsinteressen, die mit der Europäischen Nachbarschaftspolitik ihre regionale Fortentwicklung fand, setzte sich nun in der UfM mit ihrer dezidiert nüchternen Projektorientierung zumindest formal fort, selbst wenn die Europäische Union zwischenzeitlich erkannt hat, ,,dass in der Praxis nicht die erwarteten Ergebnisse erzielt werden konnten“.62

Die gegenwärtigen Entwicklungen in Nordafrika stellen europäische Außenpolitik vor die zentrale Herausforderung einer umfassenden Strategiedebatte. So versuchte man durch die UfM eine weniger politisierte Ebene der Kooperation - auch in Anerkennung vermeintlich stabiler autoritärer Systeme im südlichen Mittelmeerraum - zu lancieren. Die jüngsten

61 Vgl. dazu auch das Eingeständnis der Kommission in einer Antwort auf eine Anfrage des Europäischen Parlaments, dass die UfM nicht in einem politischen Vakuum zu lokalisieren sei. Vgl. Reply to oral question H0359/10 by Mrs Kratsa-Tsagaropoulou, 01.09.2010, Dok. P7_RH(2010)0359.

62 Europäische Kommission/Hohe Vertreterin der Europäischen Union für Außen- und Sicherheitspolitik: Gemeinsame Mitteilung an den Europäischen Rat, das Europäische Parlament, den Wirtschafts- und Sozialausschuss und den Ausschuss der Regionen. Eine Partnerschaft mit dem südlichen Mittelmeerraum für Demokratie und gemeinsamen Wohlstand, KOM (2011) 200, S. 13. 
Ereignisse in Tunesien, Libyen und Ägypten zeichnen aber eher ein Bild der nachhaltigen Politisierung der Kooperation. Auch nötigten diese die Europäische Union inzwischen dazu, das Versäumnis eines angemessenen Einbezugs der politischen Dimension der Beziehungen und die Vernachlässigung der Demokratieförderung einzugestehen, ${ }^{63}$ woraus sie mittlerweile die Notwendigkeit des Aufbaus einer ,neue[n] Partnerschaft mit der Region“64 ableitet. So bleiben grundsätzliche Fragen, ob die UfM auch den veränderten Bedingungen genügt oder ob strukturelle Geburtsfehler der UfM zu einer kompletten Neuformulierung führen, in deren Verlauf die UfM lediglich noch als „Katalysator“65 fungiert. All das ist zum gegenwärtigen Zeitpunkt im Frühjahr 2011 nicht zu beantworten, da es sich um einen ergebnisoffenen Transformationsprozess in den vom , Arabischen Frühling ' erfassten Ländern des südlichen Mittelmeerraums handelt, der die politische Landkarte in Europas südlicher Nachbarschaft neu ordnet. Insofern sind gegenwärtig noch keine Rückschlüsse auf das zu erwartende Ergebnis und ipso facto für die europäische Mittelmeerpolitik möglich.

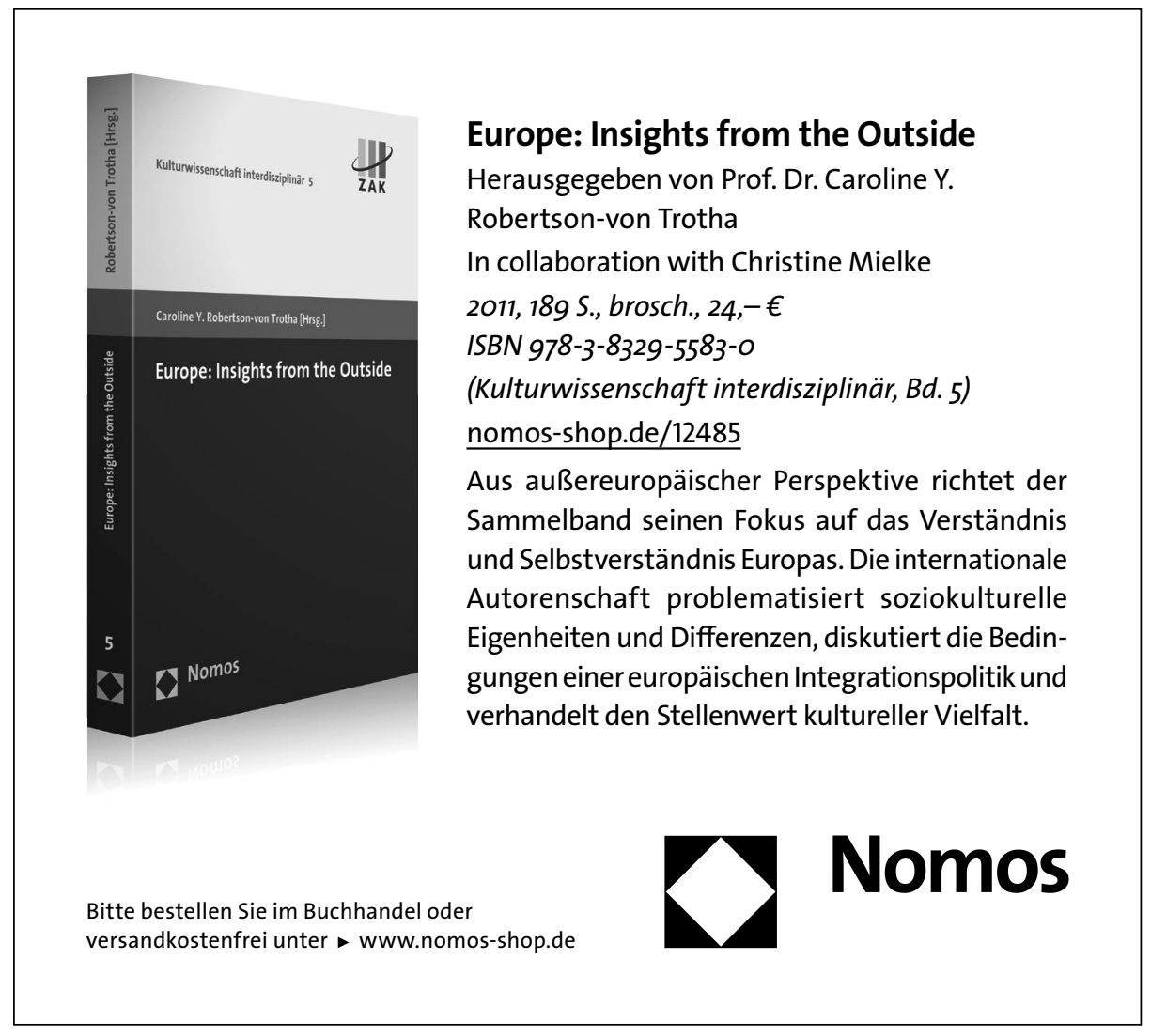

63 Vgl. Europäischer Rat: Außerordentliche Tagung des Europäischen Rates, 2011, S. 6.

64 Rat der Europäischen Union: Europäischer Rat 24./25. März 2011. Schlussfolgerungen, EUCO 10/11, S. 9.

65 Vgl. Europäische Kommission/Hohe Vertreterin der Europäischen Union für Außen- und Sicherheitspolitik: Eine Partnerschaft mit dem südlichen Mittelmeerraum für Demokratie und gemeinsamen Wohlstand, 2011, S. 13. 